\title{
La place des mathématiques dans les écoles d'ingénieurs: L'exemple français du 18e siècle jusqu'en 1920
}

Pierre Lamandé

\section{Résumé}

L'article examine l'évolution de la place des mathématiques dans les écoles d'ingénieurs depuis le dix-huitième siècle jusqu'en 1920. Au delà des particularités de la situation française, les questions posées, mais souvent occultées, sont universelles et dépassent les arguments polémiques, souvent primaires, employés par les protagonistes: place respective au niveau épistémologique, des mathématiques et des sciences "dures", influence majeure des demandes industrielles, mais aussi des situations politiques. La volonté de développer le statut social de l'ingénieur occupe également souvent une place centrale.

Mots-clés: ingénieurs, mathématiques, France, épistémologie, industrie, politique.

\begin{abstract}
This paper examines the changing role of mathematics in engineering schools since the eighteenth century until 1920. Beyond the specifics of the French situation, issues which are raised, but often ignored, are universa. They transcend the polemical arguments - frequently elementary ones -of the protagonists. They concern the respective roles of mathematics and "hard" sciences at an epistemological level, the major influence of industrial applications, and also political situations. The desire to emphasize the social status of engineers often also holds a central place.
\end{abstract}

Keywords: Engineers, mathematics, France, epistemology, industry, politics. 


\section{Introduction}

La place des mathématiques dans les enseignements scientifiques et plus particulièrement dans les formations d'ingénieurs, est une question polémique en France (et ailleurs, mais nous n'aborderons ici que le cadre hexagonal) depuis les années 1800 et elle est encore objet de confrontations entre positions antagonistes. Les arguments développés en faveur ou contre cette discipline sont d'ailleurs presque toujours les mêmes. Nous voudrions aborder ici ce problème, non sous l'angle, ô combien crucial, de l'analyse épistémologique de leur importance dans les sciences et les techniques, mais en montrant, à travers I'histoire, que ce débat fut (et est encore) souvent un reflet de luttes de pouvoir dans les institutions éducatives, économiques, techniques ou politiques. Le lien entre le diplôme et la position sociale de celui qui le détient repose en effet sur un consensus, toujours provisoire, entre les formateurs et les responsables, publics ou privés, qui sanctionnent le recrutement et la carrière des ingénieurs et donc leur place dans le monde social. Du coup, la lutte pour développer tel ou tel type de formation, pour faire reconnaître son importance et asseoir sa notoriété, n'est que très rarement sereine, appuyée sur des analyses scientifiques ou politico-économiques à long terme, mais représente presque toujours un enjeu où s'affrontent nombre de réseaux économiques, sociaux ou politiques.

\section{Mathématiques et écoles techniques supérieures au 18e siècle}

À l'origine du système français actuel, on trouve les écoles techniques fondées sous l'Ancien Régime. Les besoins de la marine en hommes compétents, notamment après le développement considérable de la flotte française entre 1660 et 1670 sous l'impulsion de Colbert, ont conduit à la création des chaires royales de mathématiques et d'hydrographie en 1669, pour former les navigateurs civils. En 1682, trois écoles des gardes de la marine se voient confier la formation des officiers de la Marine Militaire (Royale). L'édit du 5 février 1720 établit cinq écoles régimentaires destinées à l'éducation des officiers et aspirants du corps de l'Artillerie. En 1744, parait le premier règlement officiel des ingénieurs du Génie et, en 1748, naît l'École du Génie de Mézières. Les ingénieurs civils d'État apparaissent également: l'École Royale des Ponts et Chaussées en 1747, l'École Royale des Ingénieurs constructeurs de vaisseaux en 1765 et l'École Royale des Mines en 1783.

Trois points sont d'ores et déjà à souligner. D'abord, ces écoles naissent sous l'impulsion de l'État, et le sceau du centralisme marque d'une manière indélébile l'apparition de l'ingénieur français qui, même civil, est reconnu par un diplôme national et non par l'industrie . Ensuite, le recrutement de ces écoles est marqué par un concours dont le but est double: s'assurer de l'adéquation entre les besoins du corps et le recrutement d'une part, de la compétence initiale et 
finale d'autre part. Un concours d'entrée est établi dès 1749 à Mézières. II s'impose en 1755 pour le Génie, et en 1786 pour la Marine. Enfin, les concours portent avant tout sur les mathématiques qui occupent aussi une place majeure dans la formation.Les raisons de cette situation sont multiples.

Tout d'abord le maître d'œuvre de ces formations, à savoir l'État, est omni présent, non seulement à leur création, dans leurs programmes et la nomination des professeurs, mais aussi dans la régulation des flux d'entrée et de sortie, qui peuvent se tarir si la royauté ne perçoit pas le besoin de nouveaux cadres techniques. Certes, si la formation des officiers des armes savantes relève du pouvoir régalien, celle des ingénieurs des Mines ou des Ponts et Chaussées renvoie au système économique. Mais les corps d'État qui les régissent ont alors pour vocation, non de diriger directement le monde des fabriques, mais d'aider à en rationaliser les conditions d'exploitation et de transport. Le statut social des ingénieurs qui représentent directement l'administration centrale ne renvoie qu'à la volonté royale ou à la gestion politique de l'économie, Le monde des fabriques, et plus largement le monde d'Ancien Régime, est bien parcouru de remises en cause des stratégies économiques suivies par le pouvoir, mais l'autorité scientifique des grands corps ne paraît guère avoir été remise en cause avant la fin du 18e siècle. La contestation la plus importante de la vision développée par les grands commis royaux est venue de la noblesse qui, refusant de perdre ses privilèges de commandement au sein de l'armée, s'est opposée, souvent avec succès, au côté méritocratique des nouvelles formations, estimant que naissance conférait compétence.

Par ailleurs, les enseignements donnés dans ces écoles mêlent étroitement théorie et pratique, pour reprendre une dichotomie anachronique. II suffit de parcourir les cours pour voir que les outils mathématiques abordés, il est vrai souvent assez élémentaires, sont pratiquement toujours immédiatement utilisés dans des exercices reproduisant des situations que les élèves auront à maîtriser. Ainsi Bézout (1730-83) rédige des traités mathématiques différents suivant les établissements auxquels ils sont destinés. Par ailleurs, le pouvoir royal vérifie très régulièrement le fonctionnement de ces formations et leur adéquation aux buts poursuivis. Enfin, assez fréquemment, les traités élaborés, dont seuls le ou les deux premiers livres représentent le savoir exigé des élèves, rassemblent l'ensemble des connaissances dans un domaine et servent de base pour la pratique des ingénieurs durant leur carrière. On assiste même à la naissance, à l'École du génie de Mézières, de la géométrie descriptive élaborée par Gaspard Monge (1746-1818) pour donner à ses étudiants un outil adéquat à la résolution de leurs problèmes techniques. Bien sûr, ceci n'empêche pas que cette formation puisse être contestée par certains, mais leurs objections relèvent moins d'une visée théorique alternative que du refus d'un travail scolaire ou d'une arrogance sociale. 
Il existe enfin un dernier facteur qui renvoie à l'histoire du développement des sciences et des techniques. Sous l'Ancien Régime, la science ne guide pas le développement économique ou industriel. Certes, elle peut se révéler utile dans de nombreux domaines, mais la première révolution industrielle n'est pas initiée par les découvertes scientifiques. II n'y a pas de théorie de la machine à vapeur, la nouvelle chimie n'est pas née, l'électricité n'en est qu'à ses premiers balbutiements etc. Bref, la physique expérimentale est encore plus un jeu savant qu'un outil de domestication de la nature. Les sciences les plus structurées sont alors considérées comme des branches des mathématiques. La division de l'étude de la physis en mathématiques pures et mixtes, physique générale et physique particulière, exposée dans le Discours préliminaire de I'Encyclopédie est significative. Le critère distinctif n'est pas le jeu spéculatif opposé à l'utilisation dans un domaine donné, mais l'existence de principes premiers d'où la science découle par déduction. Les mathématiques sont le domaine où l'abstraction des principes premiers a été possible. Les mathématiques pures sont des spéculations sur la quantité et l'étendue figurée, premières caractéristiques sensibles des corps. En ajoutant l'impénétrabilité et le mouvement, on aborde les lois de la mécanique et l'on rentre dans les mathématiques mixtes (mécanique, astronomie géométrique, optique, acoustique, pneumatique auxquels est ajoutée l'analyse des hasards). Quand la propriété générale ou principe premier d'un domaine du savoir nous échappe, ce qui est le cas de la physique générale comme de la physique particulière, la seule ressource qui nous reste, dit Jean le Rond d'Alembert (1717-83), c'est d'amasser le plus de faits possible, et de les disposer dans l'ordre le plus naturel. On voit que nous sommes loin des concepts d'application, de théorie et de pratique, et que l'une des idées force de la révolution scientifique du 17e siècle, la mathématisation de la physis qui permet de dérouler le savoir déductivement à partir de vérités premières, est toujours non seulement présente mais utilisée dans la classification du savoir. La répartition des classes de l'Académie des Sciences, comme l'ouverture intellectuelle de la plupart des savants montrent bien qu'il y a une volonté d'unification des divers domaines scientifiques sur des principes qui trouvent leur modèle dans les mathématiques.

\section{Le début du $19 \mathrm{e}$ siècle}

\subsection{L'École Polytechnique: un modèle et ses remises en question}

Si l'École Polytechnique rompt avec certaines des traditions d'Ancien Régime, notamment en unifiant la formation initiale des ingénieurs de l'État, tant civils que militaires et en augmentant considérablement leurs effectifs qui passent de quelques dizaines à quatre cents, elle en reprend d'autres .Le concours d'entrée, garant du mérite, subsiste et reste avant tout mathématique. Cette unification avait pour but essentiel de donner à l'ensemble des responsables du système technique un langage commun jugé indispensable, tant au niveau politique que scientifique. Les 
mathématiques, dont la géométrie descriptive est devenue partie intégrante, apparaissent alors comme cette langue commune de la science et des corps techniques.

Mais l'École Polytechnique a aussi, dans l'esprit de ses fondateurs, outre le devoir de former les cadres techniques de l'État, vocation à créer la science, ce qu'elle fait au moins jusque dans les années 1850, mais le discours perdure. Elle a cependant construit un modèle spécifique, celui d'une école qui tire moins son prestige social de son aura scientifique que de ses liens avec l'État. C'est à cette tradition que l'on doit la dichotomie entre l'ingénieur d'État et l'ingénieur civil, entre ingénieur encyclopédiste destiné aux plus hautes fonctions et ingénieur spécialisé . De là vont naître les polémiques et les débats sur les contenus disciplinaires.

\subsubsection{L'école de Monge}

Gaspard Monge (1746-1818) est l'homme clé de la fondation de Polytechnique. Pour lui, plus que la classification du savoir héritée du $18 \mathrm{e}$ siècle, c'est le principe d'application qui hiérarchise les sciences et les arts et justifie l'organisation du cursus de l'école. La diversité de l'art de l'ingénieur est, dans son optique, réduite à l'application de quelques savoirs généraux, ce qui permet de concilier les différentes missions de l'école. La méthode pédagogique repose sur une association étroite de la théorie (cours magistraux) et de son application pratique dans les petites classes. La géométrie descriptive ou stéréotomie, qui occupe au départ la première place, trouve à s'appliquer à la coupe des pierres et des bois, au dessin, à la cartographie, aux machines, à l'architecture et à la fortification. La nouvelle théorie chimique illustrée par Lavoisier est enseignée et mise en application dans les laboratoires. Indépendante des corps de l'État, l'École est vue comme la matrice devant fournir l'ensemble des cadres scientifiques de la Nation.

L'économie du projet de Monge est remise en cause au bout d'un an par la création des écoles d'application dépendant des corps d'ingénieurs de l'État. Cette modification est la conséquence de l'abandon de tout plan général d'Instruction Publique: I'enseignement primaire est négligé, les écoles professionnelles abandonnées, l'École Normale fermée et l'enseignement supérieur est réduit aux écoles spéciales (droit et médecine). Le projet encyclopédique de l'École Polytechnique est rejeté et les écoles spéciales prévues pour les ingénieurs sont transformées en écoles d'application qui reprennent le modèle des anciennes écoles d'ingénieurs et sont réservées à ceux qui sortent de Polytechnique. L'école des Mines de Paris, celle des Ponts et Chaussées, les écoles d'application militaire renaissent . Le coup d'État de Brumaire sauve le privilège exclusif, mais le contrôle de l'institution passe désormais par un compromis avec les corps d'ingénieurs. 


\subsubsection{De l'École de Monge à l'École de Laplace}

À mesure que les écoles d'application, tant civiles que militaires, s'organisent, elles réclament la suppression des cours spécialisés de l'École qu'elles pensent devoir leur revenir. Ainsi, dès 1797, les leçons de fortification, de travaux civils et d'architecture décorative sont transférés, soit à l'École du Génie de Metz, soit à celle des Ponts et Chaussées. Amputée de ces applications, la géométrie descriptive est réduite progressivement à sa technique géométrique et à ses applications à la coupe des pierres et des bois ainsi qu'au dessin des ombres. La chimie qui occupait une place importante et originale dans le cursus initial de l'École est aussi affectée. Non qu'elle risque d'être démembrée, car seule l'École des Mines s'intéresse à la chimie appliquée, mais sa faible importance dans le concours de sortie la marginalise. En effet, à la suite de l'apparition des écoles d'application, un classement est établi à la sortie de l'École dès 1806 qui détermine le choix du corps. Les élèves se concentrent donc sur les matières les plus importantes pour cet examen de sortie.

La contrepartie du déclin de la géométrie descriptive et de la chimie est la montée des mathématiques et plus précisément de l'analyse et de la mécanique rationnelle. Lagrange (17361813), qui est initialement chargé de ces cours, leur donne dès le départ une orientation très théorique correspondant à sa vision du savoir. Son programme est en effet de réduire toute la mécanique et la géométrie à l'analyse, et sa réalisation passe par une réflexion approfondie sur les principes. Avec l'appui de Laplace (1744-1827), l'École établit au bout de quelques années un programme d'analyse beaucoup plus développé et cette matière devient le cours principal de l'École dès le début des années 1800. La réorganisation de 1816, à l'initiative de Laplace, renforce cette emprise.

Les écoles d'application ont très vite contesté l'aspect trop abstrait que prenait l'enseignement de l'École, à mesure qu'elle laissait aux écoles d'application les parties les plus pratiques de la formation.Ainsi, en 1811, la Commission des Fortifications écrit.

"Le comité considère en premier lieu, la détermination des programmes et demande. $1^{\circ}$ Que, dans les démonstrations, la synthèse soit employée concurremment avec l'analyse, spécialement dans tous les cas où elle donne des solutions plus simples et des formules plus commodes dans la pratique. $2^{\circ}$ Que, dans les formules, on ne néglige pas de donner, à côté des solutions et des formules rigoureuses, les méthodes approximatives qu'il est indispensable ou commode d'employer dans les services publics. $3^{\circ} Q u^{\prime}$ à la suite des solutions, on développe les principales applications des formules ou des méthodes et les modifications qu'elles doivent subir pour devenir applicables».

On retrouve ici un débat appelé à une longue postérité: l'analyse comme modèle d'abstraction versus la géométrie comme modèle de mathématique concrète, non seulement par 
ses applications immédiates, mais aussi et surtout par l'intuition qui peut soutenir ses raisonnements. Le débat parcourt aussi l'École. C'est dans ses cours à Polytechnique, au début des années 1820, que Cauchy (1789-1857) présente les bases actuelles de l'analyse mathématique, et son enseignement fut contesté comme trop abstrait, tant par les élèves que par la direction et les écoles d'application. Les questions posées à cette époque, indépendamment du problème pédagogique, sont toujours actuelles. Comment l'enseignement doit-il évoluer en fonction des nouvelles théories scientifiques? Comment savoir si des connaissances, jugés un moment abstraites, ne seront pas à la base des pratiques futures, y compris dans le domaine des applications? Peut-on faire évoluer l'enseignement supérieur sans réformer l'enseignement secondaire qui le precede ? Les besoins du présent ne cachent-ils pas ceux de l'avenir ? En 1839, Coriolis (1792-1843), directeur des études écrit dans un rapport.

"La société aujourd'hui n'a plus pour ainsi dire le temps d'étudier la nature pour l'admirer ni le loisir de chercher la vérité pour en faire l'objet de ses contemplations [...] Elle demande aux sciences qu'elle répand pour l'éducation publique des applications aux diverses industries; elle veut que leur enseignement soit dirigé vers l'accroissement de son bien-être, en servant aux perfectionnements des arts utiles $"$.

En 1848 et 1849 Polytechnique fait l'objet d'une violente campagne de la part des ingénieurs civils, puissamment relayée à l'Assemblée et au gouvernement par la majorité conservatrice. Son enseignement est jugé excessivement abstrait, inutile pour l'ingénieur et dangereux pour la société. Au delà de ce vernis rhétorique, on assiste alors à un combat mené par les ingénieurs civils pour obtenir un statut social analogue à celui des ingénieurs d'État, qui trouve un relais dans une classe politique offusquée par les positions républicaines de nombre d'élèves de l'école de la rue Descartes.

\subsubsection{Les réformes du second $19 \mathrm{e}$ siècle}

La réforme conduite par Le Verrier (1811-77) en 1851 leur donne en partie satisfaction: l'esprit de l'enseignement polytechnicien devra être désormais essentiellement pratique, le seul critère étant l'utilité pour les applications, et tout développement de pure théorie est écarté. Deux décennies plus tard, l'aspect utilitariste est cependant remis en question, tant par le développement des sciences que par la volonté de conserver à l'École son rôle de formation scientifique. La défaite de 1870 qui a montré aux yeux des contemporains la supériorité de la science allemande, a permis, ici comme dans l'Université, aux défenseurs de la science pure 
d'impulser des réformes essentielles mises en place sous la Troisième République. Camille Jordan (1838-1922), dans une lettre de 1880, l'explique bien.

«En ce qui concerne l'enseignement à l'intérieur de l'École, une grande latitude a été donnée aux professeurs depuis quelques années. Mr. Hermite a usé de cette faculté pour apporter à l'enseignement de l'analyse des modifications profondes que les progrès de la science rendaient, à mon avis, nécessaires. En effet, si l'École doit, avant tout, satisfaire aux exigences des services publics, il ne faut pas perdre de vue qu'elle absorbe la grande majorité des jeunes gens doués de l'aptitude mathématique; elle nuirait aux progrès des sciences si elle ne dirigeait pas son enseignement de manière à leur fournir les notions fondamentales dont ils auraient besoin plus tard pour se livrer à l'étude plus approfondie des mathématiques ».

\subsection{L'apparition de l'ingénieur civil: l'École des mineurs de Saint Étienne et Centrale}

Le développement de la science et du monde industriel introduit, durant le premier 19e siècle, des évolutions majeures dans le rapport entre sciences pures et productions techniques. $\mathrm{Si}$, à l'orée du siècle, les découvertes chimiques permettent un début de production industrielle, la métallurgie française se développe sans grand support théorique, sur l'imitation du modèle anglais, et il en est de même pour bien d'autres secteurs. La science est toujours loin de pouvoir toujours guider le processus industriel. Surtout, le processus technique est non théorisé et la science de l'ingénieur civil reste à créer. On réalise que la réflexion scientifique et la modélisation mathématique des phénomènes physiques à laquelle elle conduit ne sont pas la seule composante du progrès technique. L'industrie civile accentue le désir de rationalisation technique et économique du processus de fabrication. C'est à cette période que s'introduit le calcul presque quotidien des coûts de revient et de la valeur ajoutée ainsi que la décomposition des postes de production. La formation de l'ingénieur se doit d'être, non seulement scientifique, mais aussi technique, voire économique. Ce type de questions est au cœur de la réflexion de l'École des mineurs de Saint Étienne et l'École Centrale .

\subsubsection{L'École des mineurs de Saint-Étienne}

Sa création en août 1816 résulte d'un débat entre le corps des Mines et Beaunier (17791835), ingénieur du corps proposé pour en être le directeur. Les protagonistes reconnaissaient l'écart entre les ingénieurs du corps des Mines et les directeurs d'exploitation et s'accordaient sur la nécessité d'une école pratique de mineurs, installée au centre d'un bassin houiller et industriel et à visée professionnelle, Mais ses liens avec l'École des Mines de Paris sont problématiques. En 
particulier, doit-on laisser à Paris le privilège de la théorie, et ne donner à Saint-Étienne que la pratique ? Les ordonnances de 1816 reconnaissent à l'École de Paris son statut d'école d'application de Polytechnique pour former les ingénieurs du corps, mais introduisent des élèves externes, futurs directeurs d'exploitation ou d'usines. L'ordonnance concernant Saint-Étienne laisse en fait Beaunier libre de mener à sa guise l'expérience éducative. Elle spécifie seulement que l'instruction des élèves, recrutés en principe parmi les enfants de mineurs, doit être au moins égale à celle reçue dans les écoles primaires. La visée initiale est bien d'y former l'encadrement inférieur de l'industrie.

Les mathématiques ne sont pas absentes. Le premier règlement spécifie que les élèves doivent maîtriser dans ce domaine " les éléments de mathématiques dont la connaissance est indispensable pour dresser les plans et mesurer les surfaces et les solides; la levée des plans superficiels et souterrains; le nivellement ; les éléments du dessin appliqué au tracé et au lavis des plans, des machines et des constructions ". Ce programme, orienté vers la pratique, était suffisamment vague pour permettre des développements ultérieurs. C'est effectivement ce qui se produisit. Le cours de mathématiques comprenait dans un premier ensemble arithmétique, géométrie, algèbre, géométrie descriptive, lever de plans souterrains et superficiels avec boussoles, planchettes et autres instruments, et dans un second des notions de mécanique. Le conseil général des Mines jugea ce programme trop théorique et impossible à assimiler par les élèves, mais il ne fut suivi, ni par le directeur des Mines, ni par l'École. Celle-ci en effet voit arriver comme élèves, non des fils d'ouvriers mineurs, mais des enfants de directeurs d'usines ayant une instruction secondaire. La direction en prend acte et augmente le niveau . Contrairement aux craintes du Conseil des Mines, les élèves furent dans l'ensemble aptes à suivre ce programme ambitieux. Certes, seule une moitié des élèves des premières promotions se voit accorder le brevet de l'école. Si le recrutement se faisait sur examen par l'ingénieur des mines du département, sans grande exigence théorique et en privilégiant l'origine professionnelle, l'école n'entendait en rien céder sur le niveau de compétence de ses diplômés. L'enseignement était de fait tiré vers le haut et les redoublements nombreux.

Cette volonté de relever le statut de la formation s'est poursuivie, se traduisant par des réformes successives qui ont toutes accru le poids des matières théoriques, dont les mathématiques. En 1831, une ordonnance établit un nouveau mode de recrutement. La voie normale devient l'admission après un double examen sur le français (une dictée) et les mathématiques .Il est subi, d'abord devant un ingénieur des mines, puis devant un jury d'examen, en l'occurrence le conseil de l'École. Les jeunes gens admissibles à Polytechnique peuvent entrer sans concours. Enfin des élèves libres ont la possibilité de suivre les cours et de préparer ainsi leur future admission. C'était dégager complètement l'entrée à l'école du lien à l'entreprise, et lui 
substituer une norme de connaissances. L'examen de sortie distinguait trois sortes de brevets. Le brevet de troisième classe exigeait de connaître en mathématiques I'arithmétique, la tenue des livres, la géométrie, la théorie des machines simples et le jeu des principales machines employées dans les mines et usines. Obtenir le brevet de première classe, le plus élevé, supposait de connaître l'arithmétique (y compris la tenue des livres), l'algèbre, la géométrie des lignes, surfaces et solides, la trigonométrie rectiligne et la levée des plans souterrains et de surface, la mécanique y compris la description des machines employées dans les mines et les usines. Cette réforme sanctionnait la réussite de l'École, mais permettait aussi de la situer par rapport à une nouvelle concurrente qui avait dans son programme des cours de mines et métallurgie: Centrale.

\subsubsection{L'École Centrale des Arts et Manufactures}

Elle est créée en 1828 par un groupe comprenant A. Lavallée (1791-1873), propriétaire et juriste, J.B. Dumas (1800-84) chimiste, T. Olivier (1793-1853) ancien polytechnicien et E. Péclet (1793-1857), ancien normalien ; elle reste privée jusqu'en 1857. Comme l'École des mineurs de Saint-Étienne, elle part du constat de la nécessité de former les acteurs de l'industrie et plus particulièrement de fonder la science industrielle. Toutes deux connaissent un succès presque immédiat dans un monde du travail avec lequel elles sont, dès l'origine, étroitement liées. Toutes deux également adaptent leur mode de formation aux spécificités françaises.Il n'était pas question en effet de reprendre le système anglais dans lequel l'industrie privée forme ses cadres; c'est dans une école, proche mais distincte du monde des fabriques, que les techniciens de l'industrie sont formés. Les raisons de cette attitude renvoient à une double réalité: le poids du modèle de l'École Polytechnique et le constat que le mode de transmission et d'élaboration du savoir technique anglais ne peut être repris en France car le tissu industriel y est différent, moins dense et moins ancien. Toutes deux enfin sont traversées par le débat sur le lien entre abstraction et pratique. Les fondateurs de Centrale refusaient qu'y soient enseignées " les théories mathématiques trop élevées, l'expérience ayant montré que ces théories sont rarement utiles dans les applications, et que, dans le cas contraire, le simple exposé des résultats obtenus par une analyse transcendante peut suffire ". . Les différences sont cependant réelles. Centrale ne dépend pas d'un corps d'État, est beaucoup plus généraliste que l'École des mineurs de Saint-Étienne et décerne le diplôme d'ingénieur à l'issue des trois années de formation.

Le recrutement des premières décennies montre des similitudes entre les deux établissements: il repose avant tout sur un réseau de relations. Saint-Étienne utilise les ingénieurs du corps des Mines et ses liens avec l'industrie locale. Centrale distribue chaque année des prospectus, tant en France qu'à l'étranger . La société d'encouragement de l'industrie nationale est mise à contribution. Les recteurs sont sollicités pour fournir des listes de candidats intéressés. Les professeurs de l'École jouent de leurs relations personnelles.Comme à Saint-Étienne, les admissibles à Polytechnique peuvent entrer sans concours.Le lien avec les classes préparatoires 
est cependant plus vite établi à Centrale qu'à Saint-Étienne. Un réseau de professeurs de mathématiques spéciales, dont certains sont chargés de faire passer l'examen d'entrée, contribue à la réputation de Centrale et, à Paris même, se constituent très vite des préparations privées, quelquefois liées à des enseignants de l'École. Elles se généralisent en province dans les années 1860. Le niveau du concours d'entrée de Centrale n'était pas au départ très élevé. J. H. Weiss note que sous la Monarchie de Juillet, seuls 10,5\% des centraliens sont bacheliers. C'est peut-être une des raisons pour lesquelles Centrale, comme Saint-Étienne, ne diplômait pas alors tous les élèves. . Il est cependant clair, bien que nous ne disposions pas de travaux suffisamment précis pour Centrale, que les exigences théoriques du concours d'entrée, y compris en mathématiques, n'ont cessé de croître au fil du temps et il faut bien constater que cette évolution s'est accompagnée d'un accroissement du taux de diplômés parmi les élèves, phénomène également observé à SaintÉtienne. En 1841, l'algèbre et la géométrie sont inclues dans le concours d'entrée.En 1877, le programme d'admission comprend en mathématiques la majeure partie des matières enseignées en mathématiques spéciales et est analogue à ceux de Centrale et de l'École des Mines de Paris. En 1879, une troisième année est introduite et le diplôme d'ingénieur couronne les études.

Même si elles sont moins dominantes qu'à Polytechnique, les mathématiques ont une place non négligeable à Centrale, en première année avec des cours de géométrie descriptive, de mécanique et d'analyse générale et en seconde année avec les applications de la géométrie descriptive . La troisième est consacrée à la science industrielle . Cet enseignement porte la marque d'Olivier qui reprochait à l'école de la rue Descartes d'avoir détourné, sous l'impulsion de Laplace, le projet initial de Monge en substituant au primat de la géométrie descriptive celui de l'analyse mathématique. S'il est vrai que la constitution d'une science industrielle, destinée à donner une vision rationnelle des problèmes scientifiques et techniques des fabriques, est au cœur des enseignements centralien et stéphanois, ces deux Écoles reconnaissent le rôle des mathématiques dans son élaboration. Leur recrutement a été, au fil du temps, de plus en plus strict quant à la culture mathématique, et cette exigence relève autant des besoins scientifiques que du désir de reconnaissance sociale.

\subsubsection{Les Écoles d'Arts et métiers: une ascension continue}

C'est en 1803 que Bonaparte décida de transformer l'ancienne école de Liancourt en pépinière de sous officiers de l'industrie . Les plus jeunes (de 7 à 13 ans) s'initiaient à la lecture, l'écriture, le calcul, à la grammaire et au dessin. Les artistes (à partir de 13 ans) se formaient à l'algèbre, la géométrie, au dessin industriel et à diverses techniques de la métallurgie, de la fonderie et du bois. Une élite d'aspirants allait plus loin en algèbre, trigonométrie, géométrie descriptive et dans l'espace, mécanique et chimie. Le programme de l'École de Chalons 
comprenait une introduction à la physique et à la chimie, à la mécanique, la géométrie appliquée, les rudiments de l'algèbre, le dessin industriel, l'arithmétique, la calligraphie et la grammaire française. Sans grands moyens, ces deux écoles sont victimes de l'oubli du gouvernement, du mépris pour les travaux mécaniques, et de la méfiance des classes laborieuses habituées à l'apprentissage et à la formation sur le tas. Sans véritable procédure ni examen d'entrée, elles recrutaient pour l'essentiel des fils de militaires qui souvent savaient à peine lire et écrire à leur arrivée. Sous l'Empire, la majorité de ceux dont on a pu retracer la carrière furent militaires ou fonctionnaires. Sous la Restauration, bien que le gouvernement n'apprécia guère ces écoles, quelques améliorations sont mises en œuvre. L'ordonnance du 26 février 1817 fixe l'âge d'entrée entre 13 et 16 ans et assure une certaine régularité des critères d'admission, Mais l'absence d'industries lourdes et la persistance de mentalités préindustrielles explique que les élèves d'avant 1840 se soient retrouvés en majorité soldats, fonctionnaires ou artisans.

La Monarchie de Juillet fut plus favorable. En 1831, les Écoles d'Arts et Métiers sont rattachées au ministère du Commerce et des Travaux Publics. La réforme de 1832 s'accompagne d'une intégration plus poussée du théorique et de la pratique. Les nouveaux programmes comprennent: rédaction française et grammaire, mécanique, physique, chimie, arithmétique, algèbre jusqu'aux équations du second degré, géométrie appliquée et descriptive, le tout orienté vers des applications pratiques . Le développement de l'enseignement primaire (loi Guizot de 1832) et la création des écoles primaires supérieures permettent une amélioration du recrutement. L'objectif de plus en plus affirmé de former des ouvriers spécialisés, des contremaîtres et dessinateurs pour les fabriques mécaniques et les besoins croissants de l'industrie et de la technique conduit en 1843 à la création d'une troisième École d'Arts et Métiers et à une insertion croissante des gadzarts dans l'univers industriel. En 1850, sur 1343 anciens élèves encore vivants, 907 (les deux tiers) travaillaient dans l'industrie et les emplois allaient d'ouvrier spécialisé, contremaître et dessinateur à directeur d'usine, chef d'entreprise et industriel à succès.

Le statut des Écoles continua de s'améliorer. En 1865, des cours supérieurs de physique et chimie sont introduits et les ateliers modernisés. Dans les années 1880, les Écoles d’Arts et Métiers sont devenues le premier fournisseur d'ingénieurs mécaniciens de France . Le décalage entre la vocation officielle de formation des sous-officiers de l'industrie et la réalité était flagrant. Leurs programmes ne dépassent pas alors le niveau élémentaire en géométrie descriptive et mécanique, même s'ils vont plus loin en algèbre. Le combat des gadzarts pour faire reconnaître leurs compétences est couronné par la réforme de 1885. Le niveau du recrutement est relevé, correspondant au programme des nouvelles écoles primaires supérieures. La géométrie analytique est introduite dans les cours des Écoles ainsi que l'hygiène industrielle, un complément d'histoire et de géographie, de français et une spécialisation des ateliers. Cette loi faisait des Écoles d'Arts et Métiers un véritable enseignement secondaire technique et l'amélioration des 
connaissances mathématiques permettait aux meilleurs de préparer avec succès le concours d'entrée à Centrale. En 1897, un enseignement complet d'électricité avec laboratoires est créé. En 1899, le niveau des programmes en sciences et mathématiques est encore relevé. L'aboutissement de ces réformes est l'attribution du diplôme d'ingénieur en 1907.

Comme pour Centrale et l'École des Mines de Saint-Étienne, la reconnaissance des qualifications données par les Écoles d'Arts et Métiers est passée par un relèvement du niveau des connaissances scientifiques et mathématiques, phénomène d'autant plus accentué durant le dernier quart du siècle que la seconde révolution industrielle liait irréversiblement sciences et techniques. On pourrait multiplier les exemples, mais tous montrent le lien entre l'élévation du niveau de l'enseignement théorique et celui du statut social des élèves .Le développement des classes préparatoires privées et publiques dans la seconde moitié du siècle répond à cette exigence.

\section{Le débat des années 1916-1920 sur les écoles d'ingénieurs}

\subsection{L'enseignement des sciences au début du $20 \mathrm{e}$ siècle}

Au début du 20e siècle, la question de l'enseignement des sciences se pose dans tous les pays industrialisés et traverse toutes les disciplines, même si nous ne parlerons que des mathématiques. En France, la réforme de 1902, qui établit quatre baccalauréats d'égale valeur, A, $B, C$ et $D$, et donne aux sciences un statut égal à celui des lettres, ne met pas fin aux réflexions sur les contenus de l'enseignement. En 1908, une Commission Internationale de l'Enseignement Mathématique est créée au Congrès International des Mathématiciens. Carlo Bourlet (18661913), I'un des responsables de la commission française du CIEM, y décrit bien les enjeux.

"L'enseignement des mathématiques, dans nos lycées, collèges et gymnases de tous pays, passe actuellement par ce que d'aucuns nomment une crise et qui n'est en somme qu'une fièvre de croissance, un malaise né de la rapidité même de l'évolution du savoir humain [...] Brusquement, les professeurs se sont trouvés placés devant ce double problème à résoudre: non seulement acquérir eux-mêmes les connaissances nouvelles au fur et à mesure de leur éclosion, mais encore les faire pénétrer dans leur enseignement ».

Il faut aussi souligner que les mathématiciens étaient clairement conscients de la liaison nécessaire entre mathématiques et sciences expérimentales, car Carlo Bourdet ajoute un peu plus loin. 
«Il ne nous est plus permis maintenant de présenter à nos élèves la science mathématique sous un aspect purement spéculatif et il nous faut coûte que coûte, plus encore pour rendre service à la société dans son ensemble qu'à chacun de nos étudiants en particulier, nous efforcer de faire plier les abstractions mathématiques aux nécessités de la réalité [...] L'industrie, fille de la science du XIXe siècle, règne aujourd'hui en maîtresse dans le monde [...] Notre devoir impérieux est donc de préparer nos jeunes gens, dont on nous a confié l'éducation, à connaître, à pratiquer et à faire progresser les sciences expérimentales où cette industrie puise ses forces. La conclusion qui en découle est inéluctable: dans nos classes secondaires, le professeur de mathématiques, soucieux, non pas d'orner les esprits de ses élèves, mais de rendre service à sa race et à l'humanité, doit résolument écarter de son enseignement tout ce qui n'aura pas une utilité plus ou moins directe dans les applications ».

C'est dans ce climat que les écoles d'ingénieurs françaises réfléchissent sur le contenu de leurs enseignements à la fin de la première guerre mondiale, en intégrant les nécessités de la reconstruction du pays. II semble important de souligner qu'il n'y a pas alors antagonisme entre mathématiciens et expérimentateurs, et que la question de la place respective de la théorie et de la pratique traverse tout le monde scientifique, mathématicien comme physicien ou chimiste.

\subsection{Un état des lieux}

On sait le développement énorme des formations techniques supérieures entre 1870 et 1914, Instituts d'Université ou fondations privées, pour la plupart vouées à des domaines spécialisés. Elle est concomitante avec le développement de l'enseignement technique secondaire. Elle correspond aussi avec la seconde révolution industrielle qui s'appuie, bien plus que la première, sur la science. Elle renvoie enfin à la prise de conscience, après la défaite de 1870, de la supériorité de l'Allemagne dans les domaines scientifiques et industriels et de la nécessité de relever les formations techniques françaises pour assurer la puissance économique et politique de la France. L'immédiate après-guerre est l'occasion d'un grand débat mené par la société des ingénieurs civils qui aborde le problème des contenus de l'enseignement (nous ne parlerons ici que la question des mathématiques), tant dans les Écoles que dans l'enseignement préparatoire. S'il n'eut pas de conséquence immédiate sur l'enseignement supérieur, il aboutit aux réformes législatives de 1919 et 1920 qui réorganisent l'enseignement technique et commercial (loi d'Astier).

Il faut revenir un instant sur la structure de l'enseignement technique supérieur de l'époque. La classification qui en est donnée par Léon Guillet (1873-1946) est importante, car elle montre les spécificités françaises et surtout influe sur l'analyse des réformes proposées. II 
distingue quatre catégories d'institutions d'enseignement technique supérieur, qui renvoient nettement à des positions sociales différenciées: $1^{\circ}$ Les grandes écoles à enseignement encyclopédique $2^{\circ}$ Les Instituts dépendants des Universités $3^{\circ}$ Les Écoles indépendantes $4^{\circ}$ Le Conservatoire National des Arts et Métiers qui occupe, de par sa libre entrée, une place très spéciale. Guillet note que la vocation encyclopédique des grandes écoles, donnant une culture technique, scientifique et en sciences humaines, a permis aux ingénieurs qui en sortaient d'occuper les places de direction dans le monde industriel. Les Écoles d'Arts et Métiers en étaient conscientes qui réclament une amélioration du niveau de culture générale afin de relever le niveau de leur qualification .

\subsection{Une spécificité française à conserver}

Si Polytechnique n'est pas mentionnée par Guillet, elle est omniprésente dans le débat. Certes les critiques anciennes envers cette École sont reprises. Le Chatelier y condamne en particulier

"la place trop importante abandonnée aux mathématiques. Le développement imprévu de la Physique et de la Chimie, l'influence tous les jours grandissante de ces deux sciences sur les progrès de l'industrie auraient exigé une évolution de l'enseignement. II n'en a rien été; la mécanique et la physique sont de plus en plus enseignées comme branches annexes des mathématiques. La méthode expérimentale, l'habitude des mesures précises sont négligées ".

Mais, si Le Chatelier veut y augmenter la place des sciences dures, les orienter dans un sens plus expérimental et pratique, il ne préconise pas pour autant d'abandonner les mathématiques qui sont pour lui une des composantes essentielles des sciences.

"L'exemple des écoles techniques montre, d'une manière évidente, que pour la préparation aux études industrielles, il faut posséder certaines grandes disciplines scientifiques: l'analyse mathématique, le calcul différentiel, la mécanique rationnelle et expérimentale, la chimie, la physique, tout particulièrement ses deux branches de la thermodynamique et de l'électricité».

Personne, en effet, ne remet en cause la nécessité d'un apprentissage mathématique, les questions portant avant tout sur sa place respective vis-à-vis de la physique et de la chimie ainsi que sur la nature de son enseignement. Quelle part respective de la théorie et de la pratique? Qui doit les enseigner? 
La comparaison avec les formations techniques des autres pays donne l'occasion à Léon Guillet, de montrer que la spécificité française est source de richesse. Analysant la formation des ingénieurs allemands, il souligne qu'ils sont très spécialisés et que cette situation pose problème. Étudiant la répartition des leçons de mathématiques suivant les diplômes, il en trouve en moyenne 600 pour les mécaniciens, 470 pour les mineurs, 530 pour les futurs topographes et 0 pour les chimistes. Il ajoute alors:

"C'est donc au moment même où la science, et par conséquent l'industrie chimique, tend de plus en plus à s'appuyer sur la science mathématique, par I'intermédiaire de la thermodynamique, c'est au moment même où les lois des équilibres chimiques viennent jeter une lumière éclatante sur certaines fabrications que, préoccupés d'une production intensive et rapide d'ingénieurs, les Hoshschulen ne permettent pas à leurs élèves de s'initier à toutes ces notions capitales, du moins sur les bases mêmes de la question».

Quant aux ingénieurs anglais, ils sont, depuis le 17e siècle jusqu'au 19e siècle, formés dans les usines mêmes. Si quelques universités comme Sheffield se sont orientées vers la pratique au 19e siècle, il faut attendre le début du 20e siècle pour qu'un véritable centre d'enseignement technique supérieur soit créé à Londres. Aux États-Unis, l'enseignement technique, dispensé dans les Universités et les Écoles Techniques, était très divers; les mathématiques y avaient une importance relativement restreinte, les laboratoires jouaient un grand rôle. Cependant une réaction contre la tendance longtemps dominante d'y singer le mode industriel s'est fait jour, au début du 20e siècle, pour introduire la théorie à l'école et laisser la pratique à l'usine.

La situation française est donc exceptionnelle: ses grandes écoles sont les seules à recruter en classes de mathématiques spéciales par voie de concours, les seules aussi à donner un enseignement encyclopédique. Cette spécificité et son histoire expliquent la place particulière des études mathématiques. Mais cette exception est manifestement pour les protagonistes du débat une source de richesse à laquelle il ne faut pas renoncer, même s'il faut l'adapter aux nouvelles conditions économiques et scientifiques.

\subsection{Quelles mathématiques?}

L'opposition récurrente entre analyse, vue comme le symbole de l'abstraction, et la géométrie, associée à l'esprit de synthèse et aux applications, réapparaît. Elle est bien illustrée par Léon Lecornu (1854-1940), professeur à l'école Polytechnique et à l'École Supérieure des Mines. S'appuyant sur l'opinion de maîtres éminents, il voudrait qu'on revienne à un enseignement plus positif. « Je connais pour ma part d'excellents professeurs de Spéciales, mais beaucoup d'autres abusent certainement des méthodes analytiques. On risque de tuer ainsi dans l'œuf cet esprit géométrique, cette intuition qui doit être la qualité dominante de l'ingénieur » . 
Après avoir critiqué l'influence germanique jugée trop abstraite (il ne parle que des mathématiques, mais l'on connaît aussi l'opposition de nombre de physiciens et chimistes françaises aux nouvelles théories physiques développées en Allemagne), il rappelle les paroles de Joseph Bertrand (1822-1900) qui, en 1848, écrivait à propos des équations de Lagrange. « La trop grande habitude de tout déduire des formules fait perdre jusqu'à un certain point le sentiment net et précis des vérités mécaniques considérées en elles mêmes. Les procédés analytiques, dont on fait aujourd'hui un si grand usage, sont plus propres à convaincre l'esprit qu'à l'éclairer » .

Ces critiques sont bien situées historiquement. On y lit en filigrane une certaine spécificité des mathématiques françaises du 19e siècle, moins spéculatives que celles d'outre-Rhin, ainsi qu'un climat anti allemand alors largement répandu. II serait bon, d'autre part, de nuancer ces interprétations des écrits des mathématiciens cités. Mais, au-delà de ces remarques, la question de fond reste bien celle du développement différencié des diverses sciences et du repli sur des savoirs bien établis aux dépens des nouveaux domaines scientifiques. Le vieil adage La nature ne fait pas de sauts est bien dans l'esprit de Lecornu et de ses confrères. L'histoire de la physique au 20 e siècle ne tardera pas à le démentir. Nous retrouvons le dilemme perpétuel de tout enseignement scientifique à visée professionnelle: comment innover, intégrer les nouveaux savoirs, à un moment où ils n'ont pas encore fait la preuve de leur efficacité dans l'exploitation des phénomènes naturels? Et cela est également valable pour les universités, a priori plus libres de leurs recherches. À l'époque où Lecornu écrit, la physique française s'engageait sur la voie du déclin, faute de renouveler le découpage hérité du19e siècle, par refus des "spéculations théoriques ", par crainte des nouvelles approches, par marginalisation des approches discontinuistes et statistiques .

\subsection{Le programme des classes de mathématiques spéciales}

La multiplicité des formations conduit Léon Guillet à distinguer deux classes d'ingénieurs, les spécialistes et le haut personnel technique. La formation des premiers demande une culture moins générale, une durée moins longue; elle peut être réservée aux instituts universitaires et à quelques écoles particulières. La formation du personnel dirigeant issu des grandes écoles réclame au contraire trois stades, à partir du baccalauréat: a) un complément important de sciences pures, notamment de mathématiques b) un enseignement général comprenant les facteurs communs à toutes les industries $\mathrm{c}$ ) un enseignement industriel complémentaire plus ou moins spécialisé. En conséquence, il juge que la formation donnée aux futurs ingénieurs entre le baccalauréat et l'entrée aux grandes écoles, assurée pour l'essentiel dans les classes préparatoires , doit avoir au moins deux buts: d'une part leur donner les bases scientifiques nécessaires à la 
poursuite de leurs études, d'autre part contribuer à la formation de toutes les qualités qui font le véritable ingénieur, notamment l'esprit d'observation et la sûreté du jugement.

"La base d'un tel enseignement doit assurément se trouver dans les sciences mathématiques. Personne ne leur conteste la plus haute importance, tant au point de vue de la formation de l'intelligence que de l'utilité future. Le seul point sur lequel de très nombreux et de très excellents esprits s'insurgent est l'abus qu'on en a fait et la façon de les enseigner».

L'enseignement des classes préparatoires ne paraît pas satisfaire ces exigences. Nombre d'élèves y restent trop longtemps, répétent le même programme et y apprennent plus les manies des examinateurs qu'un véritable savoir scientifique. De plus, elles préparent en même temps à l'École Normale Supérieure et aux Écoles d'ingénieurs, alors que la recherche et le monde technique ne réclament pas les mêmes compétences. II propose donc l'établissement de classes préparatoires spécifiques pour Normale Supérieure. Quant aux autres, elles ne doivent pas s'écarter de leur but: préparer à la carrière d'ingénieur. Elles doivent comprendre ce qui constitue les connaissances indispensables à la profession et permet au futur élève de passer aisément à travers tout l'enseignement des grandes écoles. "II faut prendre pour base de l'enseignement mathématique ce qui peut être utile dans toutes les carrières d'ingénieurs, ce que nous appellerons plus loin, pour les Sciences Industrielles, les facteurs communs ".Il y serait donc moins étendu mais plus approfondi. Ces classes doivent donner à l'élève les notions susceptibles de lui faire toucher les réalités de la vie, et enfin écarter ce qui peut le conduire à croire que tout problème industriel se met en équation et que la science de l'ingénieur n'est que mathématique. Craignant que cette réforme soit impossible dans le cadre des classes préparatoires, Guillet envisage même leur suppression et leur remplacement par des cours préparatoires, donnés dans les écoles qui feraient le tri à l'issue d'une année. Non seulement les défauts des concours seraient ainsi atténués, mais l'enseignement serait plus facilement adapté aux besoins du monde technique que dans les lycées. Cette proposition ne fut pas retenue, mais c'est bien l'idée des classes préparatoires intégrées qui était en germe. La première verra le jour à I'INSA de Lyon après la seconde guerre mondiale.

II n'y eut pas de débat sur le contenu même de l'enseignement mathématique dans les écoles d'ingénieurs. La vision largement partagée était que les mathématiques devaient, pour l'essentiel, être acquises avant l'entrée, seuls des compléments spécifiques s'insérant dans les cursus d'ingénieur. Leur diversité ne permettait pas des conclusions valables pour toutes les formations. L'urgence de la reconstruction du pays impose de former des cadres techniques immédiatement utilisables. Pour les mathématiques comme pour toutes les sciences, les enseignants et le personnel des Écoles, doivent être choisis parmi les ingénieurs mêlés effectivement et réellement au métier dans la spécialité qu'ils enseignent. Ils ne peuvent donner une formation couvrant tous les champs du savoir, et Guillet reconnaît que les progrès de la 
science peuvent conduire à de nouvelles techniques. II estime que l'ingénieur devra alors se former par lui-même, dans les Universités, dans des établissements spécifiques comme le CNAM ou dans les ouvrages spéciaux.

Reprenant une expérience séculaire, ce débat, révélateur des positions du monde des ingénieurs civils manifeste bien la double perspective qui traverse l'expérience française de formation des ingénieurs: recherche d'une adéquation du contenu aux développements de la science et de l'industrie, mais aussi légitimation de leur compétence qui passe par une maîtrise de l'abstraction.

\section{Conclusion}

Ce trop bref aperçu montre bien que la place des mathématiques dans les formations d'ingénieurs n'est pas une donnée immuable. Si elle est dominante au 18e siècle, elle le doit avant tout par une adéquation entre l'état des savoirs et les volontés de l'État. Elle est remise en question dès le début du 19e siècle, et cela n'est pas sans lien avec la montée en puissance en France d'une industrie qui a ses exigences propres. Les arguments alors développés ne changeront pas fondamentalement par la suite, même s'ils sont illustrés par des exemples différents.Ses détracteurs évoquent l'abstraction stérile opposée aux applications nécessaires à I'industrie, les difficultés d'apprentissage etc. À l'opposé, ses défenseurs s'appuient sur le lien étroit entre technique et savoir savant où les mathématiques occupent une place centrale, au moins dans certains domaines. Les illustrations données montrent que la question dépasse en réalité la situation française et ses spécificités et doit être située historiquement.

Tout d'abord, l'épistémologie des sciences révèle qu'elles ont leur vie propre. À des phases où l'esprit créatif ne s'embarrasse guère d'abstraction et privilégie l'intuition ou un formalisme aveugle pour reprendre le terme de Leibniz, succèdent des périodes où il devient nécessaire de revenir sur les bases des savoirs, ne serait-ce que la pratique expérimentale, industrielle ou non, offre des phénomènes incompréhensibles dans les cadres de pensée anciens. L'histoire montre aussi que les avancées de la science pure ont des répercussions sur la technique, et réciproquement que les problèmes expérimentaux influent sur le questionnement scientifique; instaurer a priori une coupure serait se fermer les voies de l'avenir.

Il est vrai que les nécessités sociales peuvent momentanément être indépendantes de l'évolution scientifique: l'exemple des exigences de la reconstruction après la Grande Guerre le montre clairement. II est normal que les réponses diffèrent au fil du temps. Mais une situation privilégiant uniquement la pratique ne doit pas être immuable, faute de quoi les techniciens formés risquent fort à terme de se trouver dans une impasse. La crispation sur des modèles 
éprouvés est certes humainement compréhensible, mais scientifiquement indéfendable, l'exemple de la physique en France après la Grande Guerre le montre bien.

Derrière les arguments avancés, se cache souvent des motifs plus ou moins avoués, tels que, nous l'avons vu, la volonté d'améliorer la situation sociale des ingénieurs de production. Or les exigences du monde économique évoluent elles aussi. Les capacités littéraires distinguaient les couches dominantes au 19e siècle. Dans les dernières décennies, l'ingénieur de production a souvent été supplanté, sur le plan social, par le commercial, voire le financier. Les écoles d'ingénieurs ont évidemment suivi cette évolution. Le débat sur la place des mathématiques se situe donc sur un autre plan. Dernier point sur lequel je n'insisterai pas, mais qui a parfois une grande importance: l'hostilité théorique envers un domaine scientifique peut reposer sur des raisons personnelles, notamment un désir de valoriser sa propre discipline au détriment des autres, attitude qui nie la fécondité mutuelle des domaines scientifiques.

\section{Bibliographie}

Belhoste Bruno La formation d'une technocratie: I'École Polytechnique et ses élèves de la Révolution au Second Empire, Paris, Belin, 2003.

Belhoste Bruno, Dahan Delmedico et Picon Antoine (dir.) La formation polytechnicienne 17941994, Paris, Dunod, 1994.

Bourlet Carlo " La pénétration réciproque des mathématiques pures et des mathématiques appliquées dans l'enseignement secondaire ", L'enseignement mathématique 12, 1910, pp. 372387.

Coray Daniel et alii (ed.) « One hundred years of l'Enseignement mathématique » L'Enseignement mathématique, monographie $n^{\circ}$ 39, Genève, 2003.

Day R. Charles Les écoles d'arts et métiers, Massachussets Institute of technology, 1987, (1991) Paris, Belin, traduction française.

Fontanon Claudine et Grelon André Les professeurs du Conservatoire National des Arts et Métiers, dictionnaire biographique, Paris, INRP et CNAM, 2 tomes, 1994,

Fourcy Ambroise Histoire de l'École Polytechnique, chez l'auteur, 1828, édition critique par Jean Dhombres, Paris, Belin, 1987.

Garçon Anne Françoise Entre l'État et l'usine. L'École des Mines de saint Étienne au XIXe siècle, Presses universitaires de Rennes, 2004.

Grelon André Les ingénieurs de la crise. Titre et profession entre les deux guerres Paris, EHESS, 1986.

Guillet Léon L'enseignement technique supérieur à l'après-guerre, Paris, Payot, 1918. 
Lamandé Pierre "Bretons et grandes écoles " dans La Bretagne des savants et des ingénieurs : 1825-1900, J. Dhombres (dir.), Rennes, Ouest France Editions, 1994, p. 262-277.

Picon Antoine L'invention de l'ingénieur moderne. L'École des Ponts et Chaussées 1747-1851, Paris, Presses de l'École des Ponts et Chaussées, 1992.

Pestre Dominique Physique et physiciens en France 1918-1940, Paris, Montreux, Éditions des Archives Contemporaines, 1984.

Shinn Terry Savoir scientifique et pouvoir social. L'École Polytechnique 1794-191, Paris, Presses de la fondation nationale des sciences politiques, 1980.

Taton René (dir.) Enseignement et diffusion des sciences au XVIIle siècle en France Paris, Hermann, 1964.

Weiss John Hubbel The mahing of technicological Man, Londres, the MIT Press, Cambridge, 1982. Woronoff Denis Histoire de l'industrie en France du XVle siècle à nos jours, Paris, Seuil, 1994. 